\title{
Regulation of Cell Polarity by Exocyst-Mediated Trafficking
}

\author{
Noemi Polgar and Ben Fogelgren \\ Department of Anatomy, Biochemistry and Physiology, John A. Burns School of Medicine, University of Hawaii \\ at Manoa, Honolulu, Hawaii 96813 \\ Correspondence: fogelgre@hawaii.edu
}

\begin{abstract}
One requirement for establishing polarity within a cell is the asymmetric trafficking of intracellular vesicles to the plasma membrane. This tightly regulated process creates spatial and temporal differences in both plasma membrane composition and the membrane-associated proteome. Asymmetric membrane trafficking is also a critical mechanism to regulate cell differentiation, signaling, and physiology. Many eukaryotic cell types use the eight-protein exocyst complex to orchestrate polarized vesicle trafficking to certain membrane locales. Members of the exocyst were originally discovered in yeast while screening for proteins required for the delivery of secretory vesicles to the budding daughter cell. The same eight exocyst genes are conserved in mammals, in which the specifics of exocyst-mediated trafficking are highly cell-type-dependent. Some exocyst members bind to certain Rab GTPases on intracellular vesicles, whereas others localize to the plasma membrane at the site of exocytosis. Assembly of the exocyst holocomplex is responsible for tethering these vesicles to the plasma membrane before their soluble $N$-ethylmaleimide-sensitive factor attachment protein receptor (SNARE)-mediated exocytosis. In this review, we will focus on the role and regulation of the exocyst complex in targeted vesicular trafficking as related to the establishment and maintenance of cellular polarity. We will contrast exocyst function in apicobasal epithelial polarity versus front-back mesenchymal polarity, and the dynamic regulation of exocyst-mediated trafficking during cell phenotype transitions.
\end{abstract}

\begin{abstract}
A symmetric membrane trafficking is a critical Amechanism by which cell polarity is established and maintained. It is becoming evident that a large variety of eukaryotic cells can use the octameric exocyst protein complex as a "Swiss army knife" to execute a diverse number of polarized trafficking processes. Members of the exocyst complex were first identified as regulators of polarized exocytosis in the budding yeast Saccharomyces cerevisiae, during a genetic screen of secretory mutants (Novick and Schek-
\end{abstract}

man 1979; Novick et al. 1980). Differential sedimentation in a density gradient enabled the identification of abnormally heavy yeast cells harboring mutations in genes critical for the budding of the daughter cell. Later, eight of the identified genes, Sec3, Sec5, Sec6, Sec8, Sec10, Sec15, Exo70, and Exo84 (also called EXOC1-8, respectively) were shown to encode proteins that copurified with each other, and this interacting complex was named the exocyst (Terbush et al. 1996; Guo et al. 1999a). This 750-

Editor: Keith E. Mostov

Additional Perspectives on Cell Polarity available at www.cshperspectives.org

Copyright (C) 2018 Cold Spring Harbor Laboratory Press; all rights reserved; doi: 10.1101/cshperspect.a031401

Cite this article as Cold Spring Harb Perspect Biol 2018;10:a031401 
N. Polgar and B. Fogelgren

$\mathrm{kDa}$ holocomplex is highly conserved throughout the eukaryotic kingdoms and null mutants of individual subunits have shown early lethality in multicellular organisms (Friedrich et al. 1997; Murthy et al. 2003; Fogelgren et al. 2015; Mizuno et al. 2015).

Studies of the molecular mechanisms of exocyst function have been aided by emerging knowledge of the exocyst's structure and assem- bly (Fig. 1). The exocyst belongs to the family of complexes associated with tethering containing helical rods (CATCHR), in which the subunits show generally low sequence homology, but have conserved helical bundles packed together into long rod-like structures (Chia and Gleeson 2014). Quick-freeze/deep-etch electron microscopy studies suggested that the exocyst subunits assemble in a side-by-side fashion,

A

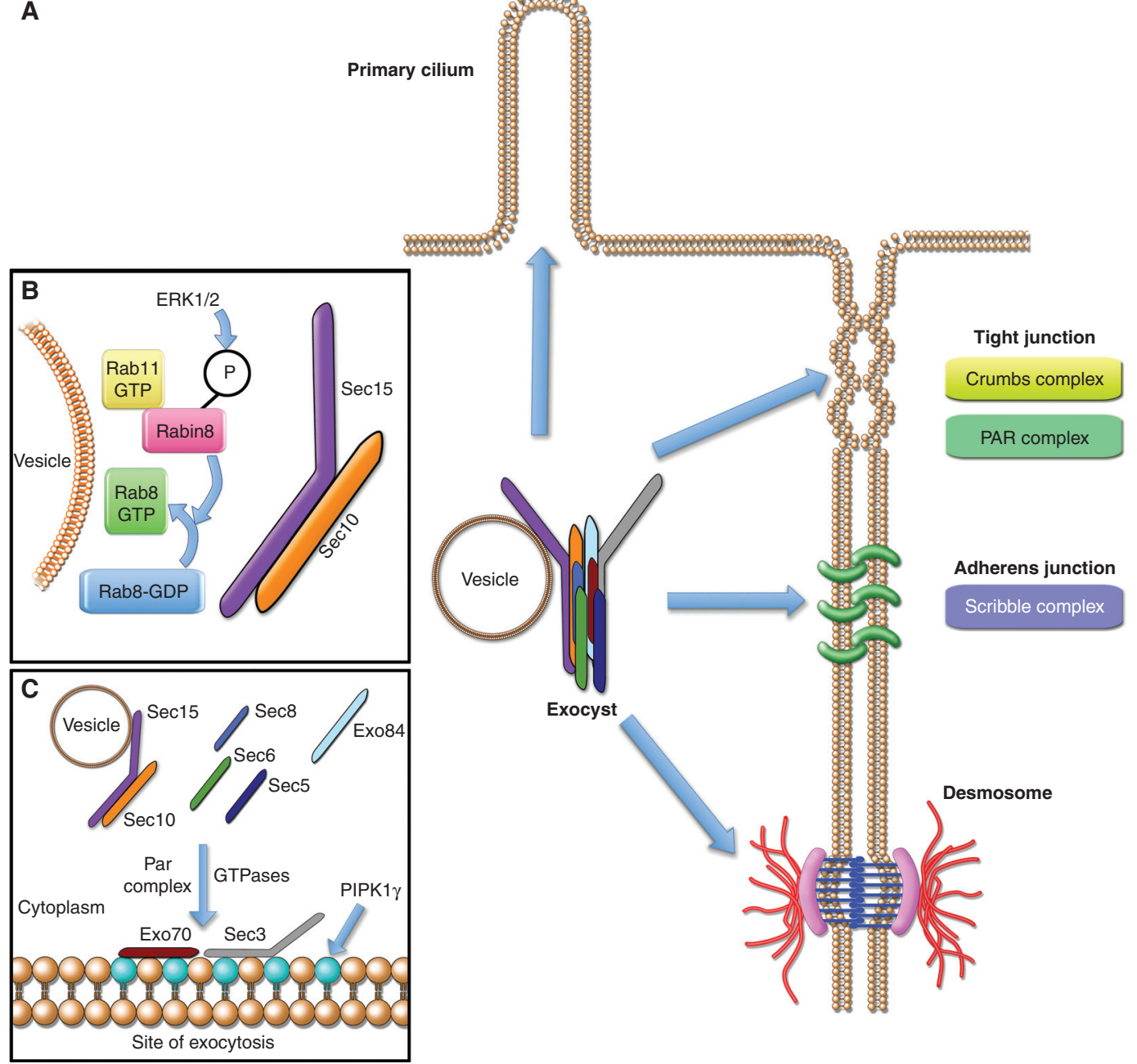

Figure 1. Exocyst function in epithelial polarity. (A) The Rab11-Rabin8-Rab8 cascade facilitates Sec15 binding to the secretory vesicle. $(B)$ PIPK $\gamma$ activity leads to a localized accumulation of the membrane phospholipid phosphatidylinositol(4,5)-bisphosphate $\left(\operatorname{Ptd} \operatorname{Ins}(4,5) P_{2}\right)$ (marked turquoise). By binding these phospholipids, Exo70 and Sec3 act as spatial landmarks for exocytosis at the plasma membrane. $(C)$ The exocyst complex regulates polarity establishment and maintenance in association with GTPases, membrane phospholipids, and polarity complexes, and by trafficking secretory vesicles to several different membrane domains of epithelial cells. 
forming a T- or Y-shaped complex, in which the amino-terminal arms are responsible for membrane tethering and regulatory interactions and the carboxy-terminal domains pack together in parallel (Hsu et al. 1998; Matern et al. 2001; Munson and Novick 2006). Early work in yeast implicated Sec15 as the subunit that directly bound Rab GTPases on the surface of secretory vesicles (Salminen and Novick 1989; Guo et al. $1999 \mathrm{~b}$ ), and Sec3 as the plasma-membranebound subunit and the spatial landmark for exocyst-destined exocytosis (Finger et al. 1998). Subunit interactions of the exocyst complex have been extensively studied using various methods in yeast and in mammals. These studies revealed and confirmed stronger pairwise interactions, such as those between Sec3Sec5, Sec6-Sec8, and Sec10-Sec15 (Guo et al. 1999a,b; Matern et al. 2001; Vega and Hsu 2001; Munson and Novick 2006; Katoh et al. 2015; Heider et al. 2016). Some exocyst interactionbased models proposed two four-subunit subcomplex-architectures for both yeast and mammalian complexes. Here, the core module of Sec3, Sec5, Sec6, and Sec8 is connected to the vesicle-attached subcomplex of Sec10, Sec15, Exo70, and Exo84 mainly through the Sec8Sec10 interaction (Katoh et al. 2015; Heider et al. 2016). This supports previous cell fractionation studies showing distinct distribution of Sec10-Exo84 and Sec5-Sec6 cofractions in rat pheochromocytoma cells (Moskalenko et al. 2003). In mammals, several of the subunits are predicted to have different isoforms as a result of alternative splicing (UniProt Consortium 2015). Discussed later in this review, alternative splicing of the exocyst genes might be a major regulatory mechanism by which cells control polarity and phenotype.

Exocyst in Trafficking and Plasma-Membrane Targeting

Initial studies in budding yeast suggested that six of the exocyst subunits traveled to the cell membrane associated with exocytic vesicles along actin cables, transported by the type $\mathrm{V}$ myosin, Myo2p (Jin et al. 2011). Unlike the rest of the exocyst, Sec3 localized to target mem- branes at the site of exocytosis independently of actin polymerization, and Exo70 could arrive to polarized sites via both actin-dependent and -independent routes (Finger et al. 1998; Boyd et al. 2004; Zajac et al. 2005; Liu and Novick 2014). Other studies of yeast and mammalian vesicle trafficking, however, suggest that the exocyst holocomplex, including Sec3 and Exo70, can be present on secretory vesicles and that the polarized subcellular localization of Sec3 is dependent on an intact secretory pathway and actin polymerization (Roumanie et al. 2005; Bendezu and Martin 2011; Bendezu et al. 2012). In addition, AP-1B, a vesicle-associated clathrin adaptor protein, which is responsible for basolateral protein sorting in epithelia, facilitated the recruitment of both Exo70 and Sec8 to the secretory vesicle (Folsch et al. 2003). This finding supports the model in which all exocyst subunits-both Exo70- and Sec8-containing subcomplexes-are present on the vesicle.

To fulfill its tethering function following vesicle delivery, the exocyst has to interact with the target membrane. This interaction is mediated through direct binding of Sec3 and Exo70 subunits with phosphatidylinositol $(4,5)$-bisphosphate $\left(\operatorname{PtdIns}(4,5) P_{2}\right)$ located primarily on the inner leaflet of the plasma membrane (He et al. 2007; Liu et al. 2007; Zhang et al. 2008; Shewan et al. 2011; Pleskot et al. 2015). The first studies of the exocyst in polarized epithelial cells implicated the exocyst in mainly basolateral vesicle trafficking to sites of cellcell contacts (Grindstaff et al. 1998; Lipschutz et al. 2000). Yet, the finding that members of the exocyst complex can directly bind with PtdIns $(4,5) P_{2}$, which can be located on the apical surface of polarized mammalian epithelial cells (Di Paolo and De Camilli 2006; GassamaDiagne et al. 2006; Martin-Belmonte et al. 2007), suggested the potential that the exocyst may also take part in apical delivery under certain conditions. Although this exocyst-phospholipid interaction may be crucial for exocytic events, the spatial and temporal control of exocyst-mediated exocytosis also hinges on regulation by known polarity complexes and various small GTPases. 
The Exocyst's Interaction with Polarity Determinants

Key factors in epithelial apicobasal and mesenchymal front-back polarity are the evolutionarily conserved polarity protein complexes: Crumbs, Par, and Scribble. In epithelial tissues, the Crumbs and the Par complexes show an apical localization at the apical junctional complex (AJC), whereas the Scribble complex is basolateral (Pieczynski and Margolis 2011; Worzfeld and Schwaninger 2016). In migrating cells, Crumbs and Par complexes are mainly located at the leading edge, but Scribble has been found at both the leading edge and the rear of migrating cells (Nelson 2009). One of the first reported functional interactions of the exocyst was with members of the Par complex. Early studies in yeast showed that cell division control protein 42 (Cdc42), the small GTPase associated with the Par complex, coordinates polarized exocyst localization (Zhang et al. 2001). Biochemical associations were shown between exocyst subunits Sec3 and Sec10 with Cdc42 in both yeast and mammalian cells (Zhang et al. 2001, 2008; Roumanie et al. 2005; Yamashita et al. 2010; Zuo et al. 2011). Later reports in mammalian cells confirmed a biochemical interaction between the exocyst and other members of the Par complex: the partitioning defective-3 (Par3) and atypical protein kinase C (aPKC) (Lalli 2009). However, subsequently, it was shown that the subunit of the Par complex that the exocyst interacted directly with was partitioning defective-6 (Par6) (Zuo et al. 2011), and this was an interaction stimulated by RalA GTPase activity (Das et al. 2014).

Exocyst localization also depends on the Crumbs polarity complex, consisting of three major proteins: Crumbs, protein associated with Lin-7 1 (PALS1), and PALS1-associated tight junction protein (PATJ). PALS1 regulates tight junction (TJ) architecture and, when depleted, it results in disrupted exocyst localization to the adherens junctions (AJs) (Wang et al. 2007; Pieczynski and Margolis 2011). On the other hand, studies of Drosophila embryonic epithelium formation suggested that Exo84 is essential for the apical localization of Crumbs
(Blankenship et al. 2007; Campbell et al. 2009). A recent study showed that Crumbs binds to Par6 in a Cdc42-dependent manner (Whitney et al. 2016), indicating that exocyst-Par complex interactions may be necessary for Crumbs trafficking. One study reported that the yeast Exo84 subunit binds with homologs of mammalian lethal giant larvae (Lgl), a member of the basolateral Scribble polarity complex (Zhang et al. 2005). However, a relationship between exocyst and Scribble complex members has not been shown in non-yeast cells. The above findings indicate that the exocyst may orchestrate the proper localization of each polarity complex during epithelial polarization.

\section{Exocyst Regulation by Small GTPases}

Throughout polarity establishment and maintenance, cells can direct spatial and temporal control of exocyst function through small intracellular signal transducers from the Rab, Ral, and Rho GTPase families. The exocyst interacts with numerous GTPases acting as their effector (Lipschutz and Mostov 2002; Munson and Novick 2006).

The Rab family of GTPases is generally responsible for directing intracellular vesicle trafficking to specific membrane locales. On the surface of yeast secretory vesicles destined for the budding daughter cell, the interaction of Sec15 and the Rab GTPase Sec4p is the first regulatory step of polarized exocytosis (Guo et al. 1999b). In various types of animal cells, evidence shows that the major Rab GTPases that use the exocyst as an effector are Rab8 (yeast Sec4p homolog) and Rab11. Both Rab8 and Rab11 were shown to directly bind Sec15 (Wu et al. 2005; Feng et al. 2012). Interestingly, a Rab11-Rab8 signaling cascade has been described. In this cascade, Rabin8, which is a Rab8 guanine exchange factor (GEF), binds exocyst Sec15 and Rab11-GTP as well (Chiba et al. 2013). Rab11, in turn, triggers Rabin8's GEF activity toward Rab8 (Wu et al. 2005; Knodler et al. 2010). Rab11's interaction with Sec15 seems to be GTP-dependent and uses Sec15's carboxy-terminal domain (Zhang et al. 2004; Wu et al. 2005). The Rab8-Rabin8-Rab11 
interactions with the exocyst define a subset of vesicles trafficking from the trans-Golgi network (TGN) and from the recycling endosomes to sites of exocyst-mediated exocytosis on the plasma membrane. One study in Drosophila reported that Sec15 also bound to other Rab GTPases, including Rab3 and Rab27, but not Rab4, Rab6, or Rab7 (Wu et al. 2005). Further studies elucidating the role of these interactions and exploring other possible regulatory Rabexocyst interactions are needed.

The Ral family of GTPases regulates a diverse array of cellular processes, and contributes to exocyst complex assembly via direct interactions with Sec5 and Exo84 (Moskalenko et al. 2002, 2003). Knockdown of RalA leads to either a decreased rate of exocyst assembly or a decreased stability of the holocomplex, and disrupted RalA-Sec5 interaction results in defective membrane protein sorting (Moskalenko et al. 2002). Because of an overlap in the RalAbinding sites for Exo84 and Sec5, these exocyst subunits bind RalA in a competitive manner (Jin et al. 2005). RalA was proposed to mediate vesicle trafficking by regulating assembly of the Sec5-containing subcomplex on the plasma membrane, and that of the vesicle-associated Exo84-containing subcomplex, before holocomplex assembly on the plasma membrane (Moskalenko et al. 2003). On the other hand, a more recent study showed that Ral GTPases were responsible for the spatial regulation of the exocyst, as reduction of RalA or RalB expression led to distinct changes in the subcellular localization of the complex, but contrary to earlier findings, did not appear to affect exocyst assembly (Spiczka and Yeaman 2008).

The Rho family of GTPases also has a diverse role in regulating cellular processes, including cell polarity and actin dynamics. Sec3 and Exo70 contribute to polarized targeting of the exocyst not only through their binding with membrane phospholipids but via interactions with members of the Rho GTPase family. Mutations of the Rhol GTPase disrupted the establishment and maintenance of polarized exocyst localization in yeast. The GTP-dependent interaction of Rho1 with the Sec3 amino-terminal domain likely ensures the recruitment of Sec 3 to membrane expansion sites in yeast. Yet disruption of the Sec3-Rho1 interaction did not result in complete exocyst mislocalization, as Sec5 and Exo70 appeared to be targeted correctly (Guo et al. 2001). Additionally, although activated forms of Rho3 have been shown to bind Exo70 (Robinson et al. 1999), Exo70 localization appears to be independent of Rho3, suggesting that there are parallel pathways targeting the exocyst subunits during cell polarization (Roumanie et al. 2005). It is possible that members of the Rho GTPase family have partially overlapping functions to regulate vesicle exocytosis acting through their shared effector, the exocyst. For example, Cdc42, another Rho GTPases that is essential in establishing cell polarity in a wide variety of cells, is also known to interact with both Sec3 and Exo70 subunits (Zhang et al. 2001; Wu et al. 2010). And whereas in yeast, $\mathrm{Cdc} 42$ competes with Rhol for binding the Sec3 amino-terminal region, the sites of Cdc42 and Rho3 interactions were mapped to different Exo70 domains (Zhang et al. 2001; Wu et al. 2010). Interestingly, Sec3 in higher eukaryotes lacks the amino-terminal region necessary for Cdc42/Rho1 binding, suggesting that Sec3 may have evolved a novel Rho1/Cdc42-binding domain, or may form the Rho-binding site in coordination with other exocyst components or proteins (Matern et al. 2001; Sakurai-Yageta et al. 2008).

\section{THE EXOCYST IN APICOBASAL POLARITY OF EPITHELIA}

\section{Exocyst and Basolateral Trafficking-The Forming of Cell Junctions}

Establishment and maintenance of the apicobasal cell polarity is essential for proper epithelial barrier and transport functions. Critical to establishing this polarity are specialized cellcell junctions, which not only function as essential components of the epithelial barrier, but importantly act to divide the cell's plasma membrane into structurally and functionally different apical and basolateral domains. There is much evidence that epithelial cells use the exocyst to regulate vesicle trafficking to these 
junctions, allowing for a dynamic regulation of junctional composition, assembly, and maintenance.

Following initial cell-cell contact of single epithelial cells, the mobile plasma-membrane pool of E-cadherin rapidly translocates to emerging contact sites (Adams et al. 1998). Ecadherin-mediated cell-cell adhesion triggers recruitment of the exocyst complex from the cytoplasm to sites of intercellular contacts, and as polarity continues to develop the exocyst complex accumulates at the AJC (Grindstaff et al. 1998; Yeaman et al. 2004). The AJC, which includes the TJs, the AJs, and the apical polarity complexes, connects the extracellular contacts between cells with the intracellular actin cytoskeleton.

In mammalian epithelial cells, it has been well established that the exocyst binds to and traffics E-cadherin (Yeaman et al. 2004; Xiong et al. 2012), as has also been shown for DEcadherin, the Drosophila E-cadherin ortholog (Langevin et al. 2005). During AJ formation, type I phosphatidylinositol-4-phosphate 5-kinase $\gamma$ (PIPKI $\gamma$ ) colocalizes and binds to E-cadherin (Akiyama et al. 2005; Ling et al. 2007), and by generating PtdIns(4,5) $P_{2}$, PIPKI $\gamma$ recruits Exo70 and Sec3 to target exocyst activity (Liu et al. 2007; Zhang et al. 2008). Although in Exo70-depleted cells E-cadherin is capable of forming dispersed intercellular contacts, the AJ fails to expand and form linear cohesive adhesions. In addition, PIPK $\gamma$ appears to strengthen the association between E-cadherin and Exo70, thus promoting the targeting of exocyst and its cargo to the forming AJ (Xiong et al. 2012).

Another factor regulating E-cadherin trafficking through modulating exocyst function is RalA GTPase. In fully polarized MDCK cells, expression of a constitutively active mutant RalA enhanced basolateral membrane delivery, including E-cadherin, but not apical membrane delivery (Shipitsin and Feig 2004). Similar expression of constitutively active RalB did not increase the basolateral delivery of E-cadherin. This divergence of RalA versus RalB regulation of the exocyst may be because of the Ral GTPases' distinct cellular localization: RalA, but not RalB, localizes to perinuclear recycling endosomes. It also may be because RalA and RalB have different exocyst-binding affinities: active RalB was shown to bind the exocyst less efficiently than RalA despite their high $(>80 \%)$ sequence homology (Shipitsin and Feig 2004). Thus, RalA activity likely plays a key role in exocyst-mediated epithelial polarity establishment.

However, not only the proper formation of the AJ, but its maintenance, is dependent on exocyst activity. Studies in Drosophila showed that a mutation of Sec5 allowed for DE-cadherin endocytosis, but led to an accumulation of DE-cadherin in the recycling endosomal compartment and a failure of DE-cadherin recycling to the AJ (Langevin et al. 2005). Recent evidence showed that cholera toxin interferes with the Rab11/exocyst-mediated E-cadherin trafficking to AJ, contributing to intestinal barrier failure in Drosophila (Guichard et al. 2013). In addition, toxins of Bacillus anthracis affect the AJ formation through decreasing Rab11 and Sec15, resulting in impaired barrier function both in Drosophila epithelium and human endothelial cells (Guichard et al. 2010).

Found only in vertebrates, TJs are the most apical components of the cell-cell junctional complexes, and are mainly composed of transmembrane proteins. TJs serve as a diffusion barrier that maintains cell-membrane polarity and create a semipermeable barrier that controls paracellular trafficking. The exocyst complex, along with members of the Par complex, localizes to the TJs of MDCK and other polarized epithelial cells (Charron et al. 2000; Lipschutz et al. 2000; Rogers et al. 2004). Ral GTPases have been shown to regulate TJ formation by using the exocyst as their effector, but RalA and RalB act on the process in an opposing manner. RalA is necessary for TJ formation through exocytosis and RalB regulates TJ composition via endocytosis, yet shRNA-mediated knockdown of either of these GTPases had no detectable effect on overall apicobasal membrane polarity ( $\mathrm{Ha}-$ zelett et al. 2011). With respect to epithelial barrier, knockdown of the Sec10 subunit by shRNA in MDCK cells appeared to not affect the cellular localization of ZO1, a TJ component (Zuo et al. 2009; Polgar et al. 2015). It has been shown that Sec10 overexpression in MDCK cells led to 
increased E-cadherin synthesis and delivery, and overall protection of epithelial barrier integrity (Park et al. 2010; Fogelgren et al. 2014). Therefore, follow-up analysis of TJ trafficking should be performed in more robust genetic knockout epithelial cells now that a conditional knockout mouse model is available (Fogelgren et al. 2015).

Exocyst trafficking is also important for the formation of another type of epithelial intercellular junction, the desmosomes, which form connections with the intermediate filaments of the cytoskeleton. In mammalian epithelial cells, Sec3 was noted to colocalize with a subset of the exocyst complexes at the desmosome (Andersen and Yeaman 2010). Sec6 also colocalized with desmosomal proteins in MDCK cells, although pools of Sec6 were also detected in a nondesmosomal localization (Inamdar et al. 2016). Knockdown of Sec3 did not result in an intracellular accumulation of desmosomal desmoglein (Dsg2) transport vesicles, but rather Dsg2 was abnormally distributed evenly on the cell surface, indicating successful exocytosis but impaired desmosome targeting. Reduced Sec3 expression thus disrupted the morphology of desmosomes, yet had no detectable effect on AJ formation (Andersen and Yeaman 2010).

\section{The Exocyst in the Apical Domain}

Establishment of epithelial polarity requires directed trafficking specifically to the apical plasma membrane. The exocyst is most widely recognized as a basolateral targeting complex, but there is growing evidence that in some epithelial cell types under certain conditions, the exocyst can also coordinate apical exocytosis. Although an early study using pulse-chase experiments in Sec10-overexpressing polarized MDCK cells showed an increase of basolateral membrane delivery of E-cadherin and not apical gp135, these cells also had a significant increase of intracellular vesicles trafficking to the apical membrane and an increased apical secretion of g80 (Lipschutz et al. 2000). Also, exocyst inhibition by function-blocking antibodies resulted in decreased basolateral, but not apical cargo delivery (Grindstaff et al. 1998). However, a later study, using an MDCK cell model of early cystogenesis (two- and three-cell stages) in 3D matrigel cultures, provided strong evidence that Rab8a used the exocyst as an effector in coordination with the Par complex to deliver podocalyxin/gp135 and other apical cargo to the apical initiation domain (Bryant et al. 2010).

Further evidence for exocyst-mediated apical targeting has been reported in urothelial progenitor cells of the urinary tract (Lee et al. 2016), in Drosophila photoreceptor epithelial cells (Beronja et al. 2005), in Xenopus neural tube epithelial cells (Ossipova et al. 2014), in placental syncytiotrophoblast (Gonzalez et al. 2014), and in renal collecting duct cells on aquaporin vesicles (Barile et al. 2005). Additionally, in human airway epithelia in a model of inflammatory airway diseases, the exocyst has been implicated in the apical secretion of mucus-component mucin 5AC (Li et al. 2015).

Recent evidence shows that exocyst also localizes to the primary cilium on the apical surface of renal epithelial cells and is pivotal in primary cilia formation and trafficking (Rogers et al. 2004; Zuo et al. 2009; Fogelgren et al. 2011; Polgar et al. 2015; Seixas et al. 2016). The primary cilium is a finger-like protrusion on the cell surface that functions as a sensory and signaling organelle, and its formation could be considered one of the final steps in epithelial apicobasal polarization. Defects in primary cilia formation and function characterize numerous pathologies, termed ciliopathies (Powles-Glover 2014). Several of the exocyst-regulating GTPases (and their GEFs) have been shown to regulate primary ciliogenesis, such as Rab8, Rabin8, Rab11, Cdc42, and its GEF TUBA (Knodler et al. 2010; Zuo et al. 2011; Feng et al. 2012; Choi et al. 2013; Baek et al. 2016), whereas disruption of exocyst function by silencing Sec10 impaired both primary cilium formation and signaling (Zuo et al. 2009; Fogelgren et al. 2011; Polgar et al. 2015).

The Exocyst in the Maintenance of Apicobasal Polarity

Although the exocyst contributes to the establishment of apicobasal polarity through its teth- 
N. Polgar and B. Fogelgren

ering function before exocytosis, it plays a role in the maintenance of this polarity by mediating endocytic and recycling events. In MDCK cells, certain exocyst subunits localized not only to early endosomes, but to transferrin-positive recycling endosomes and Rab11a-positive apical recycling endosomes. Blocking exocyst function using Sec8 antibodies revealed that the exocyst is necessary for basolateral and apical recycling as well as basolateral-to-apical transcytosis (Oztan et al. 2007). Knockdown of Rab11 or Sec15 resulted in a significant decrease of exocytic events of recycling GFP-fused transferrin receptor (Takahashi et al. 2012). Moreover, a fluorescently tagged Sec8 was found to travel to the cell membrane on recycling vesicles, and Sec8 knockdown led to a recycling block with a perinuclear accumulation of transferrin cargo (Rivera-Molina and Toomre 2013). Very recently, the exocyst was identified as a network hub linking key components of exo- and endocytic pathways (Jose et al. 2015). This is in support of previous data showing exocyst colocalization with different endosomal compartments (Sommer et al. 2005) and with clathrin and its adaptors in Drosophila (Langevin et al. 2005).

\section{THE EXOCYST IN FRONT-REAR POLARITY OF MIGRATORY CELLS}

Exocyst and Membrane Polarization during Cell Migration

Directional cell migration is dependent on the formation of front-rear polarity (Fig. 2). Signals from the extracellular matrix and growth factors are relayed by intracellular signaling to trigger reorganization of the plasma membrane, the cytoskeleton, and protein trafficking, which are all necessary for front-rear polarity formation. Numerous signaling pathways and trafficking mechanims necessary for the establishment for apicobasal polarity are also used to regulate front-rear polarization during cell migration. In the following sections, we will present an overview of how the exocyst contributes to front-rear polarity through plasma membrane reorganization, interactions with cell po- larity proteins and the cytoskeleton, and its role in polarized intracellular trafficking.

As is the case for apicobasal polarity of epithelial cells, one of the factors that define the front-rear polarity of migrating cells is the asymmetric distribution of membrane phospholipids. A spatiotemporal generation and enrichment of PtdIns $(4,5) P_{2}$ and phosphatidylinositol-3,4,5-triphosphate $\left(\operatorname{PtdIns}(3,4,5) P_{3}\right)$ characterizes the plasma membrane of the leading edge of migrating cells (Funamoto et al. 2002; Nelson 2009). As previously mentioned, Sec3 and Exo70 interact with the phospholipid PtdIns $(4,5) P_{2}$, and this binding is required for targeted exocyst-mediated exocytosis (He et al. 2007; Zhang et al. 2008). PIPKI $\gamma$, a type 1 phosphatidylinositol-phosphate kinase, regulates exocyst-mediated $\alpha 5$ - and $\beta 1$-integrin trafficking to the leading edge of migrating cells by generating PtdIns $(4,5) P_{2}$ and binding exocyst subunits (Thapa and Anderson 2012; Thapa et al. 2012). Knockdown of different exocyst components led to decreased integrin delivery to the cell's leading edge, hindering cell migration (Zuo et al. 2006; Spiczka and Yeaman 2008; Thapa and Anderson 2012; Thapa et al. 2012). This suggests that exocyst function directly contributes to the mechanics of cell migration.

\section{Exocyst-Mediated Cytoskeletal Polarization}

Front-rear polarization of migratory cells, and cell migration itself, involve dynamic changes in the actin cytoskeletal structure. Budding yeast mutants of the late secretory pathway, including exocyst members Sec10 and Sec15, had disorganized actin cytoskeletons. This was the first indication that the exocyst might affect actin organization (Aronov and Gerst 2004). Supporting these results, the mammalian Exo70 was shown to interact with Arpc1B, a member of the Arp2/3 complex, responsible for nucleating actin for the generation of the actin network necessary for cell migration. Exo70 acts as a downstream effector for Cdc42 and Rac1 GTPases in this respect, stimulating the actin branching necessary for filopodia and lamellipodia formation (Zuo et al. 2006; Liu et al. 
Cell Polarity and Exocyst-Mediated Trafficking
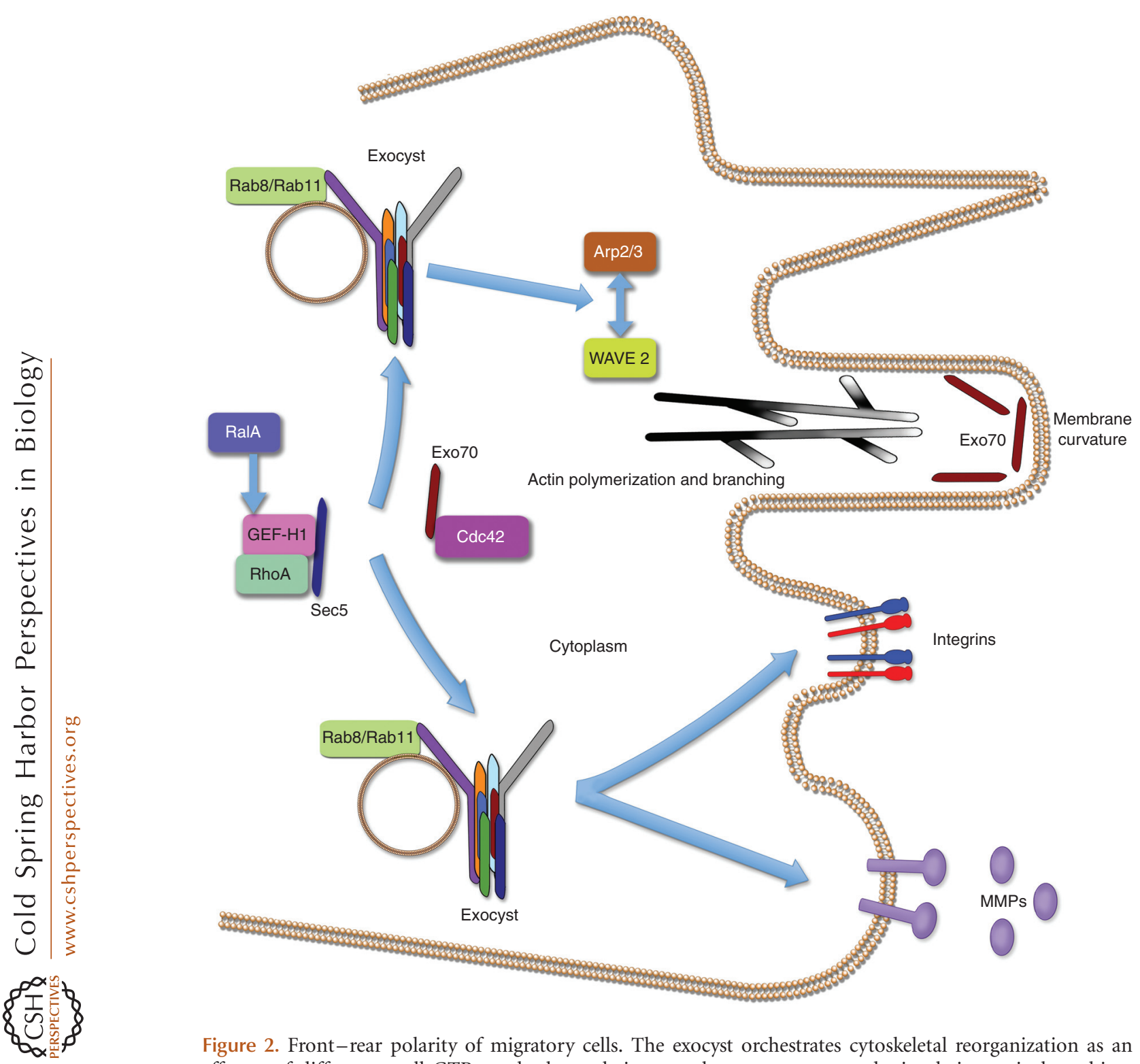

Figure 2. Front-rear polarity of migratory cells. The exocyst orchestrates cytoskeletal reorganization as an effector of different small GTPases, both regulating membrane curvature and stimulating actin branching. Additionally, evidence shows the migratory cell directs the exocyst to the leading edge to participate in integrin trafficking and signaling as well as matrix metalloproteinase (MMP) secretion. Figures were prepared using the Biomedical-PPT-Toolkit-Suite (Motifolio, Sykesville, MD).

2012). Recent evidence revealed that the exocyst subunits Exo70 and Sec6 bound with members of the wave regulatory complex (WRC), another effector of Rac1 GTPase. When this biochemical interaction was disrupted in HEK293 cells, their speed of cell motility was decreased, and the exocyst proved to regulate WRC targeting to the cell's leading edge (Biondini et al. 2016). Additionally, Exo70 contributes to Arp2/3 activity and filopodia formation by generating a curvature in the plasma membrane to make space for the forming actin network (Zhao et al. 2013). The Arp2/3-exocyst interaction was shown to be regulated by external stimulat- 
ing factors, such as epidermal growth factor (EGF) in migrating HeLa cells (Zuo et al. 2006). Interestingly, Exo70 was shown to be a direct substrate of the extracellular signal-regulated kinases (ERK1/2), and Exo70 phosphorylation by ERK1/2 appears to promote exocyst complex assembly on EGF signaling (Ren and Guo 2012). In polarized renal epithelium, increased exocyst activity by Sec10 overexpression was linked to an increased ligand-induced EGF receptor (EGFR) endocytosis and downstream signaling activity that promoted exocyst assembly (Fogelgren et al. 2014). It is therefore possible that a similar positive feedback loop exists in migratory cells, contributing to the actin cytoskeleton reorganization and the motile phenotype.

Actin organization in mammalian cells is also dependent of the Ral GTPases. RalA interactions with Sec5 and Exo84 have proved a key to actin cytoskeleton regulation. When these RalA-exocyst interactions are perturbed, cell morphological changes are observed in migratory cells, including defects in lamellipodia formation (Hazelett and Yeaman 2012). Furthermore, inhibition of RalA-Sec5 binding prevented filopodia formation triggered by inflammatory cytokine signaling (Sugihara et al. 2002). In contrast to mesenchymal cells, in normal rat kidney epithelial cells the exocyst was shown to promote cell migration as an RalB, not an RalA, effector. In this model, knockdown of RalB and Sec5, but not RalA, hindered cell motility during wound healing. RalB GTPase was necessary for both exocyst localizations to the leading edge and for the assembly of the holocomplex (Rosse et al. 2006). These results highlight that the exocyst might contribute to the migratory phenotype downstream from different regulatory signals in mesenchymal versus epithelial cells.

Coordinated modulations of microtubules and intermediate filaments are well known to regulate front-rear cell polarization and the migratory process itself (Kaverina and Straube 2011; Chung et al. 2013). At the level of intermediate filaments, knockdown of Sec8 reduced cytokeratin 8 phosphorylation, and subsequently suppressed cell migration in human oral squamous cell carcinoma cells (Tanaka and Iino 2015). The exocyst complex colocalizes with microtubules and the microtubule-organizing center in the PC12 cell line, derived from an undifferentiated pheochromocytoma (Vega and Hsu 2001). The microtubule-associated guanosine exchange factor GEF-H1 directly binds with exocyst Sec5. This binding is promoted by RalA, and leads to subsequent activation of RhoA, which is necessary for exocyst complex assembly and proper localization to the leading edge (Pathak et al. 2012).

\section{Polarized Intracellular Trafficking and the Exocyst in Migratory Cells}

Another aspect of front-rear orientation is the polarization of endo- and exocytic pathways, regulating the plasma membrane proteome distribution in the migrating cell. Recent reports showed that the exocyst is necessary for the polarized exocytosis of matrix metalloproteinases (MMPs), a family of enzymes necessary for extracellular matrix degradation, aiding cell migration and invasion. Exocyst subunits Sec3 and Sec8 were shown to directly bind IQGAP1, a Rho GTPase effector, which regulates cell polarity during migration (Noritake et al. 2005). This association was stimulated by GTP-bound Cdc42 and RhoA GTPases. In addition, invadopodial matrix degradation was affected by disruption of this interaction, as depletion of either Sec8 or IQGAP1 led to decreased exocytosis, but not biosynthesis of membrane-type MMP1 (Sakurai-Yageta et al. 2008). Further evidence showed that blocking exocyst function through Exo70, Exo84, or Sec8 knockdown abolishes, whereas Exo70 overexpression promotes, MMP secretion. Moreover, blocking Exo70 phosphorylation by Erk1/2 in tumor cells inhibits secretion of MMPs (Ren and Guo 2012). The exocyst's interaction with the endosomal Arp2/3 activator Wiskott-Aldrich syndrome protein and Scar homolog (WASH) also regulates focal delivery of MT1-MMP in invasive breast carcinoma cells (Liu et al. 2009; Monteiro et al. 2013; Yamamoto et al. 2013).

Showing subcellular polarization, the exocyst was found to accumulate at focal complexes 
forming at the leading edge, but not at focal complexes located elsewhere on migrating cells (Zuo et al. 2006; Spiczka and Yeaman 2008). This association between the exocyst and paxillin-containing focal complexes is regulated by the RalA and RalB GTPases, as Ral binding to Sec5 is necessary for the exocyst-paxillin interaction in cells (Spiczka and Yeaman 2008). The exocyst also takes part in the regulation of paxillin phosphorylation. One study in migrating rat kidney epithelial cells showed that the exocyst and the Par complex member aPKC localized to the leading edge in a mutually dependent manner (Rosse et al. 2009), and aPKC bound to the exocyst via the protein Kibra. This aPKCKibra-exocyst complex is increased during cell migration, and at the leading edge it activates JNK kinase responsible for phosphorylating paxillin and thus controlling the stability of focal adhesions (Rosse et al. 2009).

In addition to studies showing that the exocyst contributes to integrin trafficking to the leading-edge cell membrane of migratory cells, there is also evidence that the exocyst helps to regulate integrin signaling. Integrins mediate anchorage-dependent cell-cycle regulation. In the absence of integrin signal, membrane raft domains that act as signaling platforms for growth factor receptor signaling are internalized (del Pozo et al. 2004). However, similar to integrin-mediated adhesion, in Ras-dependent cancers, active RalA, Arf6 GTPase, and the exocyst promote the exocystosis of raft microdomains, contributing to anchorage-independent signaling (Pawar et al. 2016).

\section{THE EXOCYST IN EPITHELIAL- MESENCHYMAL TRANSITION}

Epithelial-mesenchymal transition (EMT) is a global process through which polarized epithelial cells undergo phenotypic changes, such as the loss of cell-cell adhesion and apicobasal polarity, and transform to acquire a motile mesenchymal state. EMT plays a role in numerous developmental processes, and is thought to play an important role in several pathologies as well, such as cancer metastasis. Until recently, nothing was known about how cells use the exocyst during the process of EMT (or the reverse, mesenchymal-epithelial transition or MET), nor what regulatory mechanisms distinguished epithelial exocyst activity from mesenchymal exocyst activity.

In 2013, the exocyst subunit Exo70 was identified as an alternative splicing target for ESRP1, one of the key epithelial-specific splicing factors to regulate isoform switching of proteins during EMT. Expression patterns revealed one Exo70 isoform to be epithelial-specific, and another alternative spliced isoform to be mesenchymal-specific, with differential expression of these isoforms correlated with cancer progression (Lu et al. 2013). Although increased expression of the epithelial-specific Exo70 isoform can induce epithelial-like properties, the mesenchymal-specific Exo70 isoform promotes lamellipodia and invadopodia formation by interacting with ARP2/3 and triggering actin branching. These results suggested that alternative splicing of the exocyst members can give rise to isoforms with distinct functions ( $\mathrm{Lu}$ et al. 2013). Cells may regulate the exocyst during EMT not only through isoform switching but by regulating the mRNA expression of mesenchymal-specific proteins, such as $\mathrm{N}$-cadherin. Recent evidence indicated that the exocyst $\mathrm{Sec} 8$ regulated $\mathrm{N}$-cadherin gene expression, but not E-cadherin, by controlling transcription of both Smad3 and Smad4 downstream from transforming growth factor $\beta$ (TGF- $\beta$ ) signaling (Tanaka et al. 2016). Given these recent discoveries, further studies are warranted to explore the differential function of exocyst isoforms, and what happens to the exocyst and associated molecules during the dynamic processes of EMT and MET.

\section{CONCLUDING REMARKS}

Research efforts in recent years have uncovered novel aspects of exocyst function and regulation in both apicobasal and front-back cell polarity. We have not only learned more about the exocyst as a tether, but gathered further evidence how the cell uses this "Swiss army knife" of a protein complex in a wide range of cellular processes necessary for polarity establishment, 
maintenance, and beyond. Yet, many more questions remain to be answered regarding transcriptional regulation of the exocyst genes, mechanisms, and regulators of holo- and subcomplex assembly, the possible role of subunit isoforms and their effect on exocyst function and regulation, as well as cargo specificity. Going forward, future studies will no doubt address these and other important questions to shed light on eukaryotic exocyst function and cell polarity.

\section{ACKNOWLEDGMENTS}

We thank Beth Lozanoff in the Department of Anatomy, Biochemistry, and Physiology at the University of Hawaii for illustrations. We thank Amanda Lee, Brent Fujimoto, and Josephine Napoli for their thoughtful feedback. Work in the Fogelgren laboratory is funded by the National Institutes of Health (Grant Nos. K01DK087852, R03DK100738, P20GM10345606A1-8293 to B.F.) and March of Dimes (Basil O'Connor Starter Scholar Research Award, Grant No. 5-FY14-56 to B.F.).

\section{REFERENCES}

Adams CL, Chen YT, Smith SJ, Nelson WJ. 1998. Mechanisms of epithelial cell-cell adhesion and cell compaction revealed by high-resolution tracking of E-cadheringreen fluorescent protein. J Cell Biol 142: 1105-1119.

Akiyama C, Shinozaki-Narikawa N, Kitazawa T, Hamakubo T, Kodama T, Shibasaki Y. 2005. Phosphatidylinositol-4phosphate 5-kinase $\gamma$ is associated with cell-cell junction in A431 epithelial cells. Cell Biol Int 29: 514-520.

Andersen NJ, Yeaman C. 2010. Sec3-containing exocyst complex is required for desmosome assembly in mammalian epithelial cells. Mol Biol Cell 21: 152-164.

Aronov S, Gerst JE. 2004. Involvement of the late secretory pathway in actin regulation and mRNA transport in yeast. J Biol Chem 279: 36962-36971.

Baek JI, Kwon SH, Zuo X, Choi SY, Kim SH, Lipschutz JH. 2016. Dynamin binding protein (Tuba) deficiency inhibits ciliogenesis and nephrogenesis in vitro and in vivo. J Biol Chem 291: 8632-8643.

Barile M, Pisitkun T, Yu MJ, Chou CL, Verbalis MJ, Shen RF, Knepper MA. 2005. Large scale protein identification in intracellular aquaporin-2 vesicles from renal inner medullary collecting duct. Mol Cell Proteom 4: 1095-1106.

Bendezu FO, Martin SG. 2011. Actin cables and the exocyst form two independent morphogenesis pathways in the fission yeast. Mol Biol Cell 22: 44-53.
Bendezu FO, Vincenzetti V, Martin SG. 2012. Fission yeast Sec3 and Exo70 are transported on actin cables and localize the exocyst complex to cell poles. PLoS ONE 7: e40248.

Beronja S, Laprise P, Papoulas O, Pellikka M, Sisson J, Tepass U. 2005. Essential function of Drosophila Sec6 in apical exocytosis of epithelial photoreceptor cells. J Cell Biol 169: 635-646.

Biondini M, Sadou-Dubourgnoux A, Paul-Gilloteaux P, Zago G, Arslanhan MD, Waharte F, Formstecher E, Hertzog M, Yu J, Guerois R, et al. 2016. Direct interaction between exocyst and Wave complexes promotes cell protrusions and motility. J Cell Sci 129: 3756-3769.

Blankenship JT, Fuller MT, Zallen JA. 2007. The Drosophila homolog of the Exo84 exocyst subunit promotes apical epithelial identity. J Cell Sci 120: 3099-3110.

Boyd C, Hughes T, Pypaert M, Novick P. 2004. Vesicles carry most exocyst subunits to exocytic sites marked by the remaining two subunits, Sec3p and Exo70p. J Cell Biol 167: 889-901.

Bryant DM, Datta A, Rodriguez-Fraticelli AE, Peranen J, Martin-Belmonte F, Mostov KE. 2010. A molecular network for de novo generation of the apical surface and lumen. Nat Cell Biol 12: 1035-1045.

Campbell K, Knust E, Skaer H. 2009. Crumbs stabilises epithelial polarity during tissue remodelling. J Cell Sci 122: 2604-2612.

Charron AJ, Nakamura S, Bacallao R, Wandinger-Ness A. 2000. Compromised cytoarchitecture and polarized trafficking in autosomal dominant polycystic kidney disease. J Cell Biol 149: 111-124.

Chia PZ, Gleeson PA. 2014. Membrane tethering. F1000Prime Rep 6: 74.

Chiba S, Amagai Y, Homma Y, Fukuda M, Mizuno K. 2013. NDR2-mediated Rabin8 phosphorylation is crucial for ciliogenesis by switching binding specificity from phosphatidylserine to Sec15. EMBO J 32: 874-885.

Choi SY, Chacon-Heszele MF, Huang L, McKenna S, Wilson FP, Zuo X, Lipschutz JH. 2013. Cdc42 deficiency causes ciliary abnormalities and cystic kidneys. J Am Soc Nephrol 24: $1435-1450$.

Chung BM, Rotty JD, Coulombe PA. 2013. Networking galore: Intermediate filaments and cell migration. Curr Opin Cell Biol 25: 600-612.

Das A, Gajendra S, Falenta K, Oudin MJ, Peschard P, Feng S, Wu B, Marshall CJ, Doherty P, Guo W, et al. 2014. RalA promotes a direct exocyst-Par6 interaction to regulate polarity in neuronal development. J Cell Sci 127: 686699.

del Pozo MA, Alderson NB, Kiosses WB, Chiang HH, Anderson RG, Schwartz MA. 2004. Integrins regulate Rac targeting by internalization of membrane domains. Science 303: 839-842.

Di Paolo G, De Camilli P. 2006. Phosphoinositides in cell regulation and membrane dynamics. Nature 443: 651657.

Feng S, Knodler A, Ren J, Zhang J, Zhang X, Hong Y, Huang S, Peranen J, Guo W. 2012. A Rab8 guanine nucleotide exchange factor-effector interaction network regulates primary ciliogenesis. J Biol Chem 287: 15602-15609. 
Finger FP, Hughes TE, Novick P. 1998. Sec3p is a spatial landmark for polarized secretion in budding yeast. Cell 92: $559-571$.

Fogelgren B, Lin SY, Zuo X, Jaffe KM, Park KM, Reichert RJ, Bell PD, Burdine RD, Lipschutz JH. 2011. The exocyst protein Sec10 interacts with Polycystin-2 and knockdown causes PKD-phenotypes. PLoS Genet 7: e1001361.

Fogelgren B, Zuo X, Buonato JM, Vasilyev A, Baek JI, Choi SY, Chacon-Heszele MF, Palmyre A, Polgar N, Drummond I, et al. 2014. Exocyst Sec10 protects renal tubule cells from injury by EGFR/MAPK activation and effects on endocytosis. Am J Physiol Renal Physiol 307: F1334F1341.

Fogelgren B, Polgar N, Lui VH, Lee AJ, Tamashiro KK, Napoli JA, Walton CB, Zuo X, Lipschutz JH. 2015. Urothelial defects from targeted inactivation of exocyst Sec10 in mice cause ureteropelvic junction obstructions. PLoS ONE 10: e0129346.

Folsch H, Pypaert M, Maday S, Pelletier L, Mellman I. 2003. The AP-1A and AP-1B clathrin adaptor complexes define biochemically and functionally distinct membrane domains. J Cell Biol 163: 351-362.

Friedrich GA, Hildebrand JD, Soriano P. 1997. The secretory protein Sec8 is required for paraxial mesoderm formation in the mouse. Dev Biol 192: 364-374.

Funamoto S, Meili R, Lee S, Parry L, Firtel RA. 2002. Spatial and temporal regulation of 3-phosphoinositides by PI 3kinase and PTEN mediates chemotaxis. Cell 109: 611623.

Gassama-Diagne A, Yu W, ter Beest M, Martin-Belmonte F, Kierbel A, Engel J, Mostov K. 2006. Phosphatidylinositol3,4,5-trisphosphate regulates the formation of the basolateral plasma membrane in epithelial cells. Nat Cell Biol 8: $963-970$.

Gonzalez IM, Ackerman WEt, Vandre DD, Robinson JM. 2014. Exocyst complex protein expression in the human placenta. Placenta 35: 442-449.

Grindstaff KK, Yeaman C, Anandasabapathy N, Hsu S, Rodriguez-Boulan R, Scheller RH, Nelson WJ. 1998. Sec6/8 complex is recruited to cell-cell contacts and specifies transport vesicle delivery to the basal-lateral membrane in epithelial cells. Cell 93: 731-740.

Guichard A, McGillivray SM, Cruz-Moreno B, van Sorge NM, Nizet V, Bier E. 2010. Anthrax toxins cooperatively inhibit endocytic recycling by the Rab11/Sec15 exocyst. Nature 467: 854-858.

Guichard A, Cruz-Moreno B, Aguilar B, van Sorge NM, Kuang J, Kurkciyan AA, Wang Z, Hang S, Pineton de Chambrun GP, McCole DF, et al. 2013. Cholera toxin disrupts barrier function by inhibiting exocyst-mediated trafficking of host proteins to intestinal cell junctions. Cell Host Microbe 14: 294-305.

Guo W, Grant A, Novick P. 1999a. Exo84p is an exocyst protein essential for secretion. J Biol Chem 274: 2355823564.

Guo W, Roth D, Walch-Solimena C, Novick P. 1999b. The exocyst is an effector for Sec4p, targeting secretory vesicles to sites of exocytosis. EMBO J 18: 1071-1080.

Guo W, Tamanoi F, Novick P. 2001. Spatial regulation of the exocyst complex by Rho1 GTPase. Nat Cell Biol 3: $353-$ 360.
Hazelett CC, Yeaman C. 2012. Sec5 and Exo84 mediate distinct aspects of RalA-dependent cell polarization. PLoS ONE 7: e39602.

Hazelett CC, Sheff D, Yeaman C. 2011. RalA and RalB differentially regulate development of epithelial tight junctions. Mol Biol Cell 22: 4787-4800.

He B, Xi F, Zhang X, Zhang J, Guo W. 2007. Exo70 interacts with phospholipids and mediates the targeting of the exocyst to the plasma membrane. EMBO J 26: 40534065.

Heider MR, Gu M, Duffy CM, Mirza AM, Marcotte LL, Walls AC, Farrall N, Hakhverdyan Z, Field MC, Rout MP, et al. 2016. Subunit connectivity, assembly determinants and architecture of the yeast exocyst complex. Nat Struct Mol Biol 23: 59-66.

Hsu SC, Hazuka CD, Roth R, Foletti DL, Heuser J, Scheller RH. 1998. Subunit composition, protein interactions, and structures of the mammalian brain sec $6 / 8$ complex and septin filaments. Neuron 20: 1111-1122.

Inamdar SM, Hsu SC, Yeaman C. 2016. Probing functional changes in exocyst configuration with monoclonal antibodies. Front Cell Dev Biol 4: 51.

Jin R, Junutula JR, Matern HT, Ervin KE, Scheller RH, Brunger AT. 2005. Exo84 and Sec5 are competitive regulatory Sec6/8 effectors to the RalA GTPase. EMBO J 24: 2064-2074.

Jin Y, Sultana A, Gandhi P, Franklin E, Hamamoto S, Khan AR, Munson M, Schekman R, Weisman LS. 2011. Myosin $\mathrm{V}$ transports secretory vesicles via a Rab GTPase cascade and interaction with the exocyst complex. Dev Cell 21: $1156-1170$.

Jose M, Tollis S, Nair D, Mitteau R, Velours C, MassoniLaporte A, Royou A, Sibarita JB, McCusker D. 2015. A quantitative imaging-based screen reveals the exocyst as a network hub connecting endocytosis and exocytosis. Mol Biol Cell 26: 2519-2534.

Katoh Y, Nozaki S, Hartanto D, Miyano R, Nakayama K. 2015. Architectures of multisubunit complexes revealed by a visible immunoprecipitation assay using fluorescent fusion proteins. J Cell Sci 128: 2351-2362.

Kaverina I, Straube A. 2011. Regulation of cell migration by dynamic microtubules. Semin Cell Dev Biol 22: 968-974.

Knodler A, Feng S, Zhang J, Zhang X, Das A, Peranen J, Guo W. 2010. Coordination of Rab8 and Rab11 in primary ciliogenesis. Proc Natl Acad Sci 107: 6346-6351.

Lalli G. 2009. RalA and the exocyst complex influence neuronal polarity through PAR-3 and aPKC. J Cell Sci 122: 1499-1506.

Langevin J, Morgan MJ, Sibarita JB, Aresta S, Murthy M, Schwarz T, Camonis J, Bellaiche Y. 2005. Drosophila exocyst components Sec5, Sec6, and Sec15 regulate DE-cadherin trafficking from recycling endosomes to the plasma membrane. Dev Cell 9: 365-376.

Lee AJ, Polgar N, Napoli JA, Lui VH, Tamashiro KK, Fujimoto BA, Thompson KS, Fogelgren B. 2016. Fibroproliferative response to urothelial failure obliterates the ureter lumen in a mouse model of prenatal congenital obstructive nephropathy. Sci Rep 6: 31137.

Li Q, Li N, Liu CY, Xu R, Kolosov VP, Perelman JM, Zhou XD. 2015. Ezrin/exocyst complex regulates mucin 5AC 
secretion induced by neutrophil elastase in human airway epithelial cells. Cell Physiol Biochem 35: 326-338.

Ling K, Bairstow SF, Carbonara C, Turbin DA, Huntsman DG, Anderson RA. 2007. Type I $\gamma$ phosphatidylinositol phosphate kinase modulates adherens junction and Ecadherin trafficking via a direct interaction with $\mu 1 \mathrm{~B}$ adaptin. J Cell Biol 176: 343-353.

Lipschutz JH, Mostov KE. 2002. Exocytosis: The many masters of the exocyst. Curr Biol 12: R212-R214.

Lipschutz JH, Guo W, O’Brien LE, Nguyen YH, Novick P, Mostov KE. 2000. Exocyst is involved in cystogenesis and tubulogenesis and acts by modulating synthesis and delivery of basolateral plasma membrane and secretory proteins. Mol Biol Cell 11: 4259-4275.

Liu D, Novick P. 2014. Bemlp contributes to secretory pathway polarization through a direct interaction with Exo70p. J Cell Biol 207: 59-72.

Liu J, Zuo X, Yue P, Guo W. 2007. Phosphatidylinositol 4,5bisphosphate mediates the targeting of the exocyst to the plasma membrane for exocytosis in mammalian cells. Mol Biol Cell 18: 4483-4492.

Liu J, Yue P, Artym VV, Mueller SC, Guo W. 2009. The role of the exocyst in matrix metalloproteinase secretion and actin dynamics during tumor cell invadopodia formation. Mol Biol Cell 20: 3763-3771.

Liu J, Zhao Y, Sun Y, He B, Yang C, Svitkina T, Goldman YE, Guo W. 2012. Exo70 stimulates the Arp2/3 complex for lamellipodia formation and directional cell migration. Curr Biol 22: 1510-1515.

Lu H, Liu J, Liu S, Zeng J, Ding D, Carstens RP, Cong Y, Xu X, Guo W. 2013. Exo70 isoform switching upon epithelialmesenchymal transition mediates cancer cell invasion. Dev Cell 27: 560-573.

Martin-Belmonte F, Gassama A, Datta A, Yu W, Rescher U, Gerke V, Mostov K. 2007. PTEN-mediated apical segregation of phosphoinositides controls epithelial morphogenesis through Cdc42. Cell 128: 383-397.

Matern HT, Yeaman C, Nelson WJ, Scheller RH. 2001. The Sec6/8 complex in mammalian cells: Characterization of mammalian Sec3, subunit interactions, and expression of subunits in polarized cells. Proc Natl Acad Sci 98: 96489653.

Mizuno S, Takami K, Daitoku Y, Tanimoto Y, Dinh TT, Mizuno-Iijima S, Hasegawa Y, Takahashi S, Sugiyama F, Yagami K. 2015. Peri-implantation lethality in mice carrying megabase-scale deletion on $5 \mathrm{qc} 3.3$ is caused by Exoc1 null mutation. Sci Rep 5: 13632.

Monteiro P, Rosse C, Castro-Castro A, Irondelle M, Lagoutte E, Paul-Gilloteaux P, Desnos C, Formstecher E, Darchen F, Perrais D, et al. 2013. Endosomal WASH and exocyst complexes control exocytosis of MT1-MMP at invadopodia. J Cell Biol 203: 1063-1079.

Moskalenko S, Henry DO, Rosse C, Mirey G, Camonis JH, White MA. 2002. The exocyst is a Ral effector complex. Nat Cell Biol 4: 66-72.

Moskalenko S, Tong C, Rosse C, Mirey G, Formstecher E, Daviet L, Camonis J, White MA. 2003. Ral GTPases regulate exocyst assembly through dual subunit interactions. J Biol Chem 278: 51743-51748.

Munson M, Novick P. 2006. The exocyst defrocked, a framework of rods revealed. Nat Struct Mol Biol 13: 577-581.
Murthy M, Garza D, Scheller RH, Schwarz TL. 2003. Mutations in the exocyst component Sec5 disrupt neuronal membrane traffic, but neurotransmitter release persists. Neuron 37: 433-447.

Nelson WJ. 2009. Remodeling epithelial cell organization: Transitions between front-rear and apical-basal polarity. Cold Spring Harb Perspect Biol 1: a000513.

Noritake J, Watanabe T, Sato K, Wang S, Kaibuchi K. 2005. IQGAP1: A key regulator of adhesion and migration. J Cell Sci 118: 2085-2092.

Novick P, Schekman R. 1979. Secretion and cell-surface growth are blocked in a temperature-sensitive mutant of Saccharomyces cerevisiae. Proc Natl Acad Sci 76: $1858-1862$.

Novick P, Field C, Schekman R. 1980. Identification of 23 complementation groups required for post-translational events in the yeast secretory pathway. Cell 21: 205-221.

Ossipova O, Kim K, Lake BB, Itoh K, Ioannou A, Sokol SY. 2014. Role of Rab11 in planar cell polarity and apical constriction during vertebrate neural tube closure. Nat Commun 5: 3734.

Oztan A, Silvis M, Weisz OA, Bradbury NA, Hsu SC, Goldenring JR, Yeaman C, Apodaca G. 2007. Exocyst requirement for endocytic traffic directed toward the apical and basolateral poles of polarized MDCK cells. Mol Biol Cell 18: 3978-3992.

Park KM, Fogelgren B, Zuo X, Kim J, Chung DC, Lipschutz JH. 2010. Exocyst Sec10 protects epithelial barrier integrity and enhances recovery following oxidative stress, by activation of the MAPK pathway. Am J Physiol Renal Physiol 298: F818-F826.

Pathak R, Delorme-Walker VD, Howell MC, Anselmo AN, White MA, Bokoch GM, Dermardirossian C. 2012. The microtubule-associated Rho activating factor GEF-H1 interacts with exocyst complex to regulate vesicle traffic. Dev Cell 23: 397-411.

Pawar A, Meier JA, Dasgupta A, Diwanji N, Deshpande N, Saxena K, Buwa N, Inchanalkar S, Schwartz MA, Balasubramanian N. 2016. Ral-Arf6 crosstalk regulates Ral dependent exocyst trafficking and anchorage independent growth signalling. Cell Signal 28: 1225-1236.

Pieczynski J, Margolis B. 2011. Protein complexes that control renal epithelial polarity. Am J Physiol Renal Physiol 300: F589-F601.

Pleskot R, Cwiklik L, Jungwirth P, Zarsky V, Potocky M. 2015. Membrane targeting of the yeast exocyst complex. Biochim Biophys Acta 1848: 1481-1489.

Polgar N, Lee AJ, Lui VH, Napoli JA, Fogelgren B. 2015. The exocyst gene Sec10 regulates renal epithelial monolayer homeostasis and apoptotic sensitivity. Am J Physiol Cell Physiol 309: C190-C201.

Powles-Glover N. 2014. Cilia and ciliopathies: Classic examples linking phenotype and genotype-An overview. Reprod Toxicol 48: 98-105.

Ren J, Guo W. 2012. ERK1/2 regulate exocytosis through direct phosphorylation of the exocyst component Exo70. Dev Cell 22: 967-978.

Rivera-Molina F, Toomre D. 2013. Live-cell imaging of exocyst links its spatiotemporal dynamics to various stages of vesicle fusion. J Cell Biol 201: 673-680. 
Robinson NG, Guo L, Imai J, Toh EA, Matsui Y, Tamanoi F. 1999. Rho3 of Saccharomyces cerevisiae, which regulates the actin cytoskeleton and exocytosis, is a GTPase which interacts with Myo2 and Exo70. Mol Cell Biol 19: 35803587.

Rogers KK, Wilson PD, Snyder RW, Zhang X, Guo W, Burrow CR, Lipschutz JH. 2004. The exocyst localizes to the primary cilium in MDCK cells. Biochem Biophys Res Commun 319: 138-143.

Rosse C, Hatzoglou A, Parrini MC, White MA, Chavrier P, Camonis J. 2006. RalB mobilizes the exocyst to drive cell migration. Mol Cell Biol 26: 727-734.

Rosse C, Formstecher E, Boeckeler K, Zhao Y, Kremerskothen J, White MD, Camonis JH, Parker PJ. 2009. An aPKC-exocyst complex controls paxillin phosphorylation and migration through localised JNK1 activation. PLoS Biol 7: e1000235.

Roumanie O, Wu H, Molk JN, Rossi G, Bloom K, Brennwald P. 2005. Rho GTPase regulation of exocytosis in yeast is independent of GTP hydrolysis and polarization of the exocyst complex. J Cell Biol 170: 583-594.

Sakurai-Yageta M, Recchi C, Le Dez G, Sibarita JB, Daviet L, Camonis J, D'Souza-Schorey C, Chavrier P. 2008. The interaction of IQGAP1 with the exocyst complex is required for tumor cell invasion downstream of $\mathrm{Cdc} 42$ and RhoA. J Cell Biol 181: 985-998.

Salminen A, Novick PJ. 1989. The Sec15 protein responds to the function of the GTP binding protein, Sec4, to control vesicular traffic in yeast. J Cell Biol 109: 1023-1036.

Seixas C, Choi SY, Polgar N, Umberger NL, East MP, Zuo X, Moreiras H, Ghossoub R, Benmerah A, Kahn RA, et al. 2016. Arl13b and the exocyst interact synergistically in ciliogenesis. Mol Biol Cell 27: 308-320.

Shewan A, Eastburn DJ, Mostov K. 2011. Phosphoinositides in cell architecture. Cold Spring Harbor Perspect Biol 3: a004796.

Shipitsin M, Feig LA. 2004. RalA but not RalB enhances polarized delivery of membrane proteins to the basolateral surface of epithelial cells. Mol Cell Biol 24: 57465756.

Sommer B, Oprins A, Rabouille C, Munro S. 2005. The exocyst component Sec5 is present on endocytic vesicles in the oocyte of Drosophila melanogaster. J Cell Biol 169: 953-963.

Spiczka KS, Yeaman C. 2008. Ral-regulated interaction between Sec5 and paxillin targets Exocyst to focal complexes during cell migration. J Cell Sci 121: 2880-2891.

Sugihara K, Asano S, Tanaka K, Iwamatsu A, Okawa K, Ohta Y. 2002. The exocyst complex binds the small GTPase RalA to mediate filopodia formation. Nat Cell Biol 4: 73-78.

Takahashi S, Kubo K, Waguri S, Yabashi A, Shin HW, Katoh Y, Nakayama K. 2012. Rab11 regulates exocytosis of recycling vesicles at the plasma membrane. J Cell Sci 125: 4049-4057.

Tanaka T, Iino M. 2015. Sec8 regulates cytokeratin8 phosphorylation and cell migration by controlling the ERK and p38 MAPK signalling pathways. Cell Signal 27: $1110-1119$.
Tanaka T, Goto K, Iino M. 2016. Sec8 modulates TGF- $\beta$ induced EMT by controlling $\mathrm{N}$-cadherin via regulation of Smad3/4. Cell Signal 29: 115-126.

Terbush DR, Maurice T, Roth D, Novick P. 1996. The exocyst is a multiprotein complex required for exocytosis in Saccharomyces cerevisiae. EMBO J 15: 6483-6494.

Thapa N, Anderson RA. 2012. PIP2 signaling, an integrator of cell polarity and vesicle trafficking in directionally migrating cells. Cell Adh Migr 6: 409-412.

Thapa N, Sun Y, Schramp M, Choi S, Ling K, Anderson RA. 2012. Phosphoinositide signaling regulates the exocyst complex and polarized integrin trafficking in directionally migrating cells. Dev Cell 22: 116-130.

UniProt Consortium. 2015. UniProt: A hub for protein information. Nucleic Acids Res 43: D204-D212.

Vega IE, Hsu SC. 2001. The exocyst complex associates with microtubules to mediate vesicle targeting and neurite outgrowth. J Neurosci 21: 3839-3848.

Wang Q, Chen XW, Margolis B. 2007. PALS1 regulates Ecadherin trafficking in mammalian epithelial cells. Mol Biol Cell 18: 874-885.

Whitney DS, Peterson FC, Kittell AW, Egner JM, Prehoda KE, Volkman BF. 2016. Binding of Crumbs to the Par-6 CRIB-PDZ module is regulated by Cdc42. Biochemistry 10: $1455-1461$.

Worzfeld T, Schwaninger M. 2016. Apicobasal polarity of brain endothelial cells. J Cereb Blood Flow Metab 36: 340-362.

Wu S, Mehta SQ, Pichaud F, Bellen HJ, Quiocho FA. 2005. Sec15 interacts with Rab11 via a novel domain and affects Rab11 localization in vivo. Nat Struct Mol Biol 12: 879885.

Wu H, Turner C, Gardner J, Temple B, Brennwald P. 2010. The Exo70 subunit of the exocyst is an effector for both $\mathrm{Cdc} 42$ and Rho3 function in polarized exocytosis. Mol Biol Cell 21: 430-442.

Xiong X, Xu Q, Huang Y, Singh RD, Anderson R, Leof E, Hu J, Ling K. 2012. An association between type I $\gamma$ PI4P 5 kinase and Exo70 directs E-cadherin clustering and epithelial polarization. Mol Biol Cell 23: 87-98.

Yamamoto A, Kasamatsu A, Ishige S, Koike K, Saito K, Kouzu Y, Koike H, Sakamoto Y, Ogawara K, Shiiba M, et al. 2013. Exocyst complex component Sec8: A presumed component in the progression of human oral squamous-cell carcinoma by secretion of matrix metalloproteinases. J Cancer Res Clin Oncol 139: $533-$ 542.

Yamashita M, Kurokawa K, Sato Y, Yamagata A, Mimura H, Yoshikawa A, Sato K, Nakano A, Fukai S. 2010. Structural basis for the Rho- and phosphoinositide-dependent localization of the exocyst subunit Sec3. Nat Struct Mol Biol 17: $180-186$.

Yeaman C, Grindstaff KK, Nelson WJ. 2004. Mechanism of recruiting Sec6/8 (exocyst) complex to the apical junctional complex during polarization of epithelial cells. J Cell Sci 117: 559-570.

Zajac A, Sun X, Zhang J, Guo W. 2005. Cyclical regulation of the exocyst and cell polarity determinants for polarized cell growth. Mol Biol Cell 16: 1500-1512.

Zhang X, Bi E, Novick P, Du L, Kozminski KG, Lipschutz JH, Guo W. 2001. Cdc42 interacts with the exocyst and reg- 
N. Polgar and B. Fogelgren

ulates polarized secretion. J Biol Chem 276: 4674546750.

Zhang XM, Ellis S, Sriratana A, Mitchell CA, Rowe T. 2004 Sec15 is an effector for the Rab11 GTPase in mammalian cells. J Biol Chem 279: 43027-43034.

Zhang X, Wang P, Gangar A, Zhang J, Brennwald P, TerBush D, Guo W. 2005. Lethal giant larvae proteins interact with the exocyst complex and are involved in polarized exocytosis. J Cell Biol 170: 273-283.

Zhang X, Orlando K, He B, Xi F, Zhang J, Zajac A, Guo W. 2008. Membrane association and functional regulation of Sec3 by phospholipids and Cdc42. J Cell Biol 180: $145-158$.
Zhao Y, Liu J, Yang C, Capraro BR, Baumgart T, Bradley RP Ramakrishnan N, Xu X, Radhakrishnan R, Svitkina T, et al. 2013. Exo70 generates membrane curvature for morphogenesis and cell migration. Dev Cell 26: 266-278.

Zuo X, Zhang J, Zhang Y, Hsu SC, Zhou D, Guo W. 2006 Exo70 interacts with the Arp2/3 complex and regulates cell migration. Nat Cell Biol 8: 1383-1388.

Zuo X, Guo W, Lipschutz JH. 2009. The exocyst protein Sec10 is necessary for primary ciliogenesis and cystogenesis in vitro. Mol Biol Cell 20: 2522-2529.

Zuo X, Fogelgren B, Lipschutz JH. 2011. The small GTPase Cdc42 is necessary for primary ciliogenesis in renal tubular epithelial cells. J Biol Chem 286: 22469-22477. 


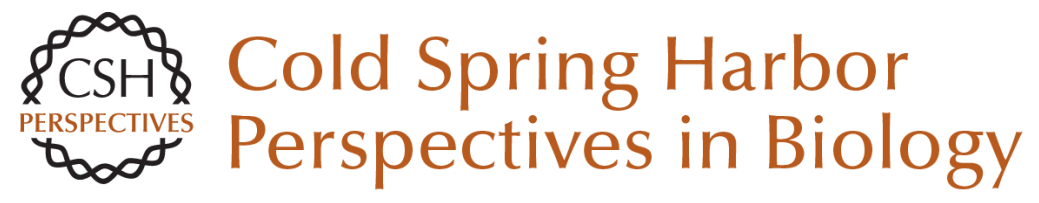

\section{Regulation of Cell Polarity by Exocyst-Mediated Trafficking}

Noemi Polgar and Ben Fogelgren

Cold Spring Harb Perspect Biol 2018; doi: 10.1101/cshperspect.a031401 originally published online March 6, 2017

\section{Subject Collection Cell Polarity}

Regulation of Cell Polarity by Exocyst-Mediated Trafficking Noemi Polgar and Ben Fogelgren

Phosphoinositides and Membrane Targeting in Cell Polarity Gerald R. Hammond and Yang Hong

Trafficking lon Transporters to the Apical Membrane of Polarized Intestinal Enterocytes Amy Christine Engevik and James R. Goldenring

Signaling Networks in Epithelial Tube Formation Ilenia Bernascone, Mariam Hachimi and Fernando Martin-Belmonte

Making Heads or Tails of It: Cell-Cell Adhesion in Cellular and Supracellular Polarity in Collective Migration Jan-Hendrik Venhuizen and Mirjam M. Zegers

Laminins in Epithelial Cell Polarization: Old Questions in Search of New Answers Karl S. Matlin, Satu-Marja Myllymäki and Aki Manninen

Epithelial Morphogenesis during Liver Development

Naoki Tanimizu and Toshihiro Mitaka

Targeting the Mucosal Barrier: How Pathogens Modulate the Cellular Polarity Network Travis R. Ruch and Joanne N. Engel
The Crumbs3 Polarity Protein Ben Margolis

Microtubule Motors in Establishment of Epithelial Cell Polarity Geri Kreitzer and Monn Monn Myat

Role of Polarity Proteins in the Generation and Organization of Apical Surface Protrusions Gerard Apodaca

Polarized Exocytosis Jingwen Zeng, Shanshan Feng, Bin Wu, et al.

Regulation of Transporters and Channels by Membrane-Trafficking Complexes in Epithelial Cells

Curtis T. Okamoto

Membrane Transport across Polarized Epithelia Maria Daniela Garcia-Castillo, Daniel J.-F. Chinnapen and Wayne I. Lencer

Mechanisms of Cell Polarity-Controlled Epithelial Homeostasis and Immunity in the Intestine Leon J. Klunder, Klaas Nico Faber, Gerard Dijkstra, et al.

The Biology of Ciliary Dynamics Kuo-Shun Hsu, Jen-Zen Chuang and Ching-Hwa Sung

For additional articles in this collection, see http://cshperspectives.cshlp.org/cgi/collection/

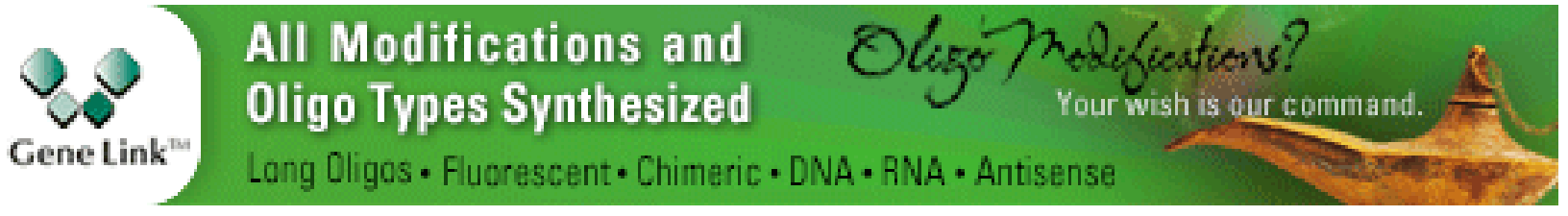

